EPiC Series in Computing
Volume 63, 2019, Pages 140-149
$\begin{gathered}\text { Proceedings of 32nd International Conference on } \\ \text { Computer Applications in Industry and Engineering }\end{gathered}$

\title{
An algorithmic approach for finding minimum spanning tree in a intuitionistic fuzzy graph
}

\author{
Kartick Mohanta ${ }^{1 *}$, Arindam Dey ${ }^{2 \dagger}$, Narayan C. Debnath ${ }^{3 \ddagger}$ and Anita Pal ${ }^{1 * \S}$ \\ 1 Department of Mathematics, National Institute of Technology, Durgapur, India. \\ km.18ma1103@phd.nitdgp.ac.in \\ 2 Department of Computer Science and Engineering, \\ Saroj Mohan Institute of Technology, Hooghly, India. \\ arindam84nit@gmail.com \\ 3 School of Computing and Information Technology, Eastern International University, Vietnam. \\ narayan.debnath@eiu.edn.vn \\ 4 Department of Mathematics, National Institute of Technology, Durgapur, India. \\ anita.buie@gmail.com
}

\begin{abstract}
The minimum spanning tree (MST) problem is a well known optimization problem in graph theory that has been used to model many real life problems, e.g., telecommunications,transportation network, routing and water supply network. The MST problems with deterministic edge costs have been worked intensively and the MST of a connected weighted graph can be determined using many efficient algorithms introduced by outstanding scientists. However, in real life scenarios, several types of uncertainties are generally encountered, because of insufficient information, imperfect information, failure or other reasons. In this paper, we concentrate on a MST problem of a undirected connected fuzzy graph in which a intuitionistic fuzzy numbers, instead of a crisp (real) number, is used to each edge as edge weight. We define this problem as intuitionistic minimum spanning tree (IMST) problem. We introduce an algorithmic approach for designing the IMST of a fuzzy. The Borůvka's algorithm is a popular greedy algorithm for designing a MST in a graph. Here, we have modified the classical Borůvka's algorithm to generate the IMST of fuzzy graph. The water distribution system is the lifeline of any city. We also describe the utility of IMST in a water distribution network. A numerical example is worked out to illustrate our proposed algorithm.
\end{abstract}

\section{Introduction}

Let $G=(V, E)$ be a a weighted undirected graph. A tree is a connected circuit less graph. A spanning tree is a subgraph of $G$ that is a tree $T=\left(V, E^{\prime}\right)$ with $E^{\prime} \subseteq E$. A minimum spanning

\footnotetext{
${ }^{*}$ Designed and implemented the manuscript

${ }^{\dagger}$ Did numerous tests and provided a lot of suggestions

¥ Contributed on developing efficient algorithm

$\S$ Guide and motivator
}

Q. Yuan, Y. Shi, L. Miller, G. Lee, G. Hu and T. Goto (eds.), CAINE 2019 (EPiC Series in Computing, vol. 63), pp. $140-149$ 
tree is a spanning tree that has the lowest possible weight. The MST is a spanning tree $T$ of graph $G$ which has the minimum cost among all the spanning tree of graph $G$. The MST problem is a fundamental and well known combinatorial optimization problem in the area of graph theory. It is widely applied in various fields of science and engineering, e.g., road network application, transportation, routing in communication channels and scheduling problems. In real life problems, edge costs are used to represent the parameter costs, capacities, demands, time, etc. Although in classical graph theory, real numbers are used in a MST problem to express the arc lengths of a connected weighted graph. We generally consider the classical MST problem as network optimization problem. In the classical MST problem, we assume the arc lengths (time, cost, distance, nature of connectivity between nodes, etc.) is certain. However, in real world problems, the information about any MST problems are generally not exact or precise due to several reasons such as incomplete data or insufficient data, less evidence, imperfect statistical. For e.g., in a weighted connected graph, the arc length may describe the traversing time between two nodes. This traveling time depends on traffic jam, accident, weather etc., each of the criteria differ from day to day. Therefore, it becomes difficult for the DM to estimate exactly the edge cost.

Several papers have been done on the Fuzzy MST [2], [3], [13], [15], [23], [24], [33], [34]. The work by Itoh and Ishii [23] is first work on this fuzzy domain and worked a MST problem with fuzzy costs as a chance constrained programming based on the necessity measure. Following that, some methods based on the ranking index for arc weights of FMST were proposed by Chang and Lee [15]. Almeida et al. [13] described the fuzzy MST problem with fuzzy costs and introduced an algorithmic method to solve this FMST. They introduced a genetic algorithm founded on fuzzy logic and probability theory to compute the FMST with fuzzy weights. In [24], they described possibility theory to describe the minimum of edge of the fuzzy graph and to find a spanning tree where the fuzzy arc weights are in fuzzy intervals. Liu [27], [28] introduced the credibility theory including pessimistic value, credibility measure and expected value as fuzzy ranking methods. Based on the credibility theory, Gao and Lu [18] studied the fuzzy quadratic minimum spanning tree problem and formulated it as expected value model, chance-constrained programming and dependent-chance programming according to different decision criteria.

In this study, we introduce an algorithmic approach for the IMST problem in intuitionistic graphs with intuitionistic fuzzy numbers, i.e., for the IMST problem. The algorithmic method is based on intuitionistic fuzzy version of the classical Borůvaa's algorithm. The Score and accuracy function of TIFN is used in the proposed algorithm for comparison between TIFN. First, we have established a water supply network using graph theory. The idea of fuzzy graph theory is used to solve this problem. We have used idea of IMST to find lowest in the case of pipeline connectivity.

Our proposed algorithmic approach has many significant advantages : (1) Compared with FMST algorithmic approach, the introduced algorithm for IMST problem is more efficient for handing the uncertainty. The ranking and the addition operation of IFN can be done easily and straight manner using mean on IFN. (2) In our IMST problem, the IMST and the cost are the most significant information for the us to use the IMST to formulate the real life scenarios. Existing approach for IMST problem can compute only the IMST of a graph. It is the main reason of this study to modify the algorithm that can determine IMST and the cost.

\section{Preliminaries}

Definition 2.1. (Intuitionistic Fuzzy set (IFS)) Let $X$ be an universal set. An IFS A is represented as 


$$
A=\left\{\frac{<T_{A}(\xi), F_{A}(\xi)>}{\xi}: 0 \leq T_{A}(\xi)+F_{A}(\xi) \leq 1, \forall \xi \in X\right\}
$$

Here $T_{A}: X \rightarrow[0,1]$ is called membership funtion and $F_{A}: X \rightarrow[0,1]$ is called non-membership function.

Definition 2.2. (Intuitionistic Fuzzy set (IFS)) Let $X$ be an universal set. An IFS A is represented as

$$
A=\left\{\frac{<T_{A}(\xi), F_{A}(\xi)>}{\xi}: 0 \leq T_{A}(\xi)+F_{A}(\xi) \leq 1, \forall \xi \in X\right\}
$$

Here $T_{A}: X \rightarrow[0,1]$ is called membership funtion and $F_{A}: X \rightarrow[0,1]$ is called non-membership function.

Definition 2.3. (Triangular Intuitionistic Fuzzy Numbers (TIFN)) If $\widetilde{A}$ be TIFN, its membershipship is defined as:

1. $T_{\widetilde{A}}(\xi)=\left\{\begin{array}{l}\left(\frac{\xi-\alpha_{1}}{\gamma-\alpha_{1}}\right) T, \text { if } \alpha_{1} \leq \xi \leq \gamma \\ \left(\frac{\alpha_{2}-\xi}{\alpha_{2}-\gamma}\right) T, \text { if } \gamma \leq \xi \leq \alpha_{2} \\ 0, \text { otherwise; }\end{array}\right.$

and its non-membership function is defined as:

2. $F_{\widetilde{A}}(\xi)=\left\{\begin{array}{l}\frac{\gamma-\xi+F\left(\xi-\beta_{1}\right)}{\gamma-\beta_{1}}, \text { if } \beta_{1} \leq \xi \leq \gamma \\ \frac{\xi-\gamma+F\left(\beta_{2}-\xi\right)}{\beta_{2}-\gamma}, \text { if } \gamma \leq \xi \leq \beta_{2} \\ 1, \text { otherwise; }\end{array}\right.$

where $0 \leq T \leq 1,0 \leq F \leq 1$, and $T+F \leq 1, \alpha_{1}, \alpha_{2}, \beta_{1}, \beta_{2}, \gamma \in \mathbb{R}$. Then $\widetilde{A}=<$ $\left(\left[\alpha_{1}, \gamma, \alpha_{2}\right], T\right),\left(\left[\beta_{1}, \gamma, \beta_{2}\right], F\right)>$ is called an intuitionistic triangular fuzzy number ITFN. For convenience ITFN $\widetilde{A}$ is written as $\widetilde{A}=\left(\left[\alpha_{1}, \gamma, \alpha_{2}\right] ; T, F\right)$ and show in figure 1 .

Definition 2.4. (Arithmetic operation between TIFN's) Let $\widetilde{A}=\left(\left[\alpha_{1}^{\widetilde{A}}, \gamma^{\widetilde{A}}, \alpha_{2}^{\widetilde{A}}\right] ; T^{\widetilde{A}}, F^{\widetilde{A}}\right)$ and $\widetilde{B}=\left(\left[\alpha_{1}^{\widetilde{B}}, \gamma^{\widetilde{B}}, \alpha_{2}^{\widetilde{B}}\right] ; T^{\widetilde{B}}, F^{\widetilde{B}}\right)$ be two TIFN's and $\lambda \geq 0$, then

1. addition of $\widetilde{A}$ and $\widetilde{B}$ is represented by $\widetilde{A} \oplus \widetilde{B}$ and defined as:

$\widetilde{A} \oplus \widetilde{B}=\left(\left[\alpha_{1}^{\widetilde{A}}+\alpha_{1}^{\widetilde{B}}, \gamma^{\widetilde{A}}+\gamma^{\widetilde{B}}, \alpha_{2}^{\widetilde{A}}+\alpha_{2}^{\widetilde{B}}\right] ; T^{\widetilde{A}}+T^{\widetilde{B}}-T^{\widetilde{A}} T^{\widetilde{B}}, F^{\widetilde{A}} F^{\widetilde{B}}\right) ;$

2. product of $\widetilde{A}$ and $\widetilde{B}$ is represented by $\widetilde{A} \cdot \widetilde{B}$ and defined as:

$$
\widetilde{A} \cdot \widetilde{B}=\left(\left[\alpha_{1}^{\widetilde{A}} \cdot \alpha_{1}^{\widetilde{B}}, \gamma^{\widetilde{A}} \cdot \gamma^{\widetilde{B}}, \alpha_{2}^{\widetilde{A}} \cdot \alpha_{2}^{\widetilde{B}}\right] ; T^{\widetilde{A}} \cdot T^{\widetilde{B}}, F^{\widetilde{A}}+F^{\widetilde{B}}-F^{\widetilde{A}} \cdot F^{\widetilde{B}}\right) ;
$$

3. multiplication by a scalar $\lambda$ with the TIFN $\widetilde{A}$ is defined as follows:

$\lambda \widetilde{A}=\left(\left[\lambda \alpha_{1}^{\widetilde{A}}, \lambda \gamma^{\widetilde{A}}, \lambda \alpha_{2}^{\widetilde{A}}\right] ; 1-\left(1-T^{\widetilde{A}}\right)^{\lambda},\left(F^{\widetilde{A}}\right)^{\lambda}\right) ;$

4. $(\widetilde{A})^{\lambda}=\left(\left[\left(\alpha_{1}^{\widetilde{A}}\right)^{\lambda},\left(\gamma^{\widetilde{A}}\right)^{\lambda},\left(\alpha_{2}^{\widetilde{A}}\right)^{\lambda}\right] ;\left(T^{\widetilde{A}}\right)^{\lambda}, 1-\left(1-F^{\widetilde{A}}\right)^{\lambda}\right)$. 


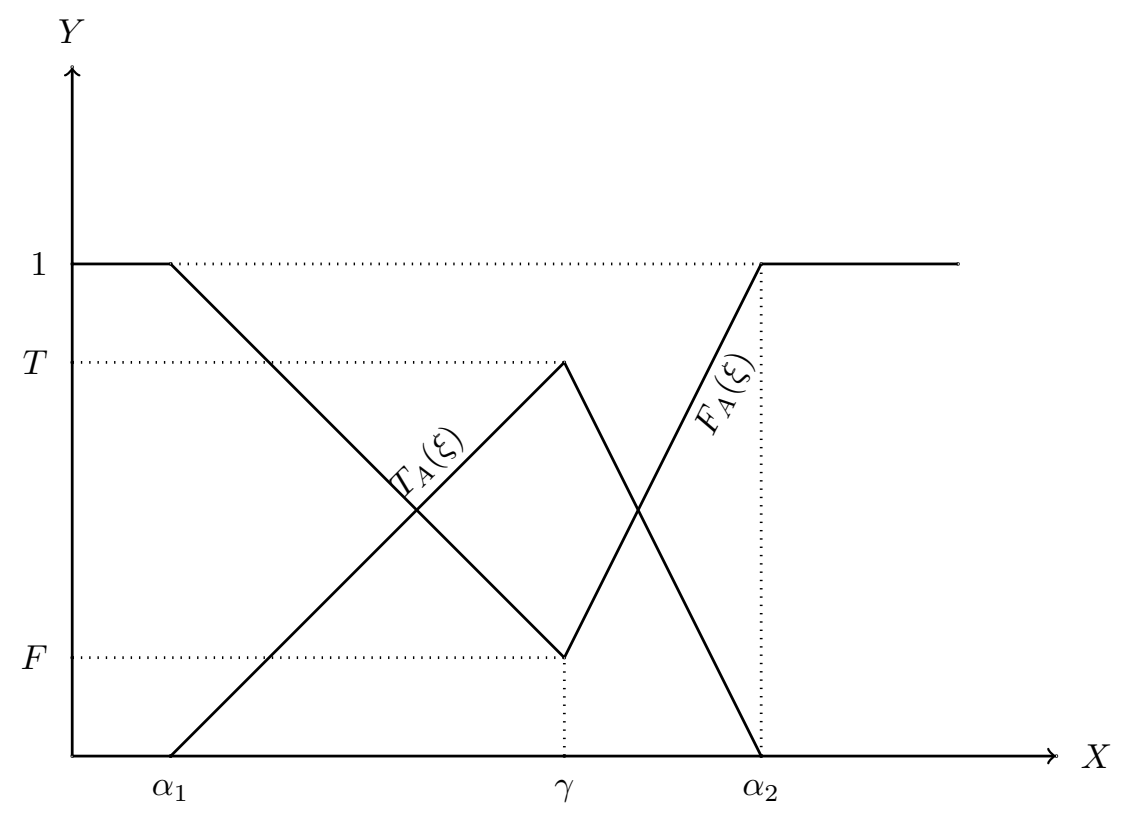

Figure 1: TIFN

Definition 2.5. (Score and accuracy function of TIFN) If $\widetilde{A}=\left(\left[\alpha_{1}^{\widetilde{A}}, \gamma^{\widetilde{A}}, \alpha_{2}^{\widetilde{A}}\right] ; T^{\widetilde{A}}, F^{\widetilde{A}}\right)$ then score function $\mathbb{S}(\widetilde{A})$ and accuracy function $\mathbb{H}(\widetilde{A})$ for $\widetilde{A}$ is defined as:

$$
\begin{gathered}
\mathbb{S}(\widetilde{A})=I(\widetilde{A}) \times\left(T^{\widetilde{A}}-F^{\widetilde{A}}\right) \\
\text { and } \mathbb{H}(\widetilde{A})=I(\widetilde{A}) \times\left(T^{\widetilde{A}}+F^{\widetilde{A}}\right)
\end{gathered}
$$

, where $I(\widetilde{A})=\frac{1}{6} \times\left(\alpha_{1}^{\widetilde{A}}+\gamma^{\widetilde{A}}+\alpha_{2}^{\widetilde{A}}\right) \times\left(1+T^{\widetilde{A}}-F^{\widetilde{A}}\right)$ Let $\widetilde{A}$ and $\widetilde{B}$ be two TIFN's, then

1. $\widetilde{A} \succ \widetilde{B}$ if $\mathbb{S}(\widetilde{A}) \succ \mathbb{S}(\widetilde{B})$;

2. $\widetilde{A} \prec \widetilde{B}$ if $\mathbb{S}(\widetilde{A}) \prec \mathbb{S}(\widetilde{B})$;

3. if $\mathbb{S}(\widetilde{A})=\mathbb{S}(\widetilde{B})$, then

(a) $\widetilde{A} \succ \widetilde{B}$ if $\mathbb{H}(\widetilde{A}) \succ \mathbb{H}(\widetilde{B})$;

(b) $\widetilde{A} \prec \widetilde{B}$ if $\mathbb{H}(\widetilde{A}) \prec \mathbb{H}(\widetilde{B})$;

(c) $\widetilde{A}=\widetilde{B}$ if $\mathbb{H}(\widetilde{A})=\mathbb{H}(\widetilde{B})$.

\section{Modified Boruvka's Algorithm for IMST}

The classical Boruvka's algorithm is a well known greedy method for finding the MST of a connected graph and the time complexity of this algorithm is $O(m \log n)$ time. The main concept of this algorithm is to cover simultaneously the arc with minimum cost adjacent on each node 
of this graph. The following variables are used in proposed algorithm.

1. A undirected triangular intuitionistic fuzzy weighted connected graph (UITFWCG) $\widetilde{G}=$ $\left(V_{\widetilde{G}}, E_{\widetilde{G}}\right)$, where $V_{\widetilde{G}}$ is the set of the vertices and $E_{\widetilde{G}}$ is the set of the edges;

2. A UITFWCG $\widetilde{G^{\prime}}=\left(V_{\widetilde{G}}, E_{\widetilde{G^{\prime}}}\right.$, where $\widetilde{G^{\prime}} \subseteq \widetilde{G}$;

3. $\xi_{i}$ is the $i$-th vertex of the vertex set $V_{\widetilde{G}}$;

4. $e_{i j}$ is the edge connecting the vertices $v_{i}$ and $v_{j}$;

5. $\widetilde{M}$ is the A triangular intuitionistic fuzzy minimum spanning tree tree of $\widetilde{G}$;

6. $\widetilde{W}\left(e_{i j}\right)$ is a discrete ITF weight associated with the edge $e_{i j}$ of $\widetilde{G}$;

7. $\widetilde{W}(\widetilde{M})$ is the ITFW of $\widetilde{M}$

\section{Proposed Bor ůvka's Algorithm:}

Input: A graph $\widetilde{G}=\left(V_{\widetilde{G}}, E_{\widetilde{G}}\right)$

Output: The resultant MST $\widetilde{M}$ and $\widetilde{W}(\widetilde{M})$

Step 1: Initialize $\widetilde{M}$ as empty graph. Set $\widetilde{G^{\prime}}=\widetilde{G}$

Step 2: for each $v_{i} \in V_{\widetilde{G^{\prime}}}$ do

Step 3: The score and accuracy function of TIFN is used for finding the sorting the edges of the graph. Based on the value of score of TIFN, we minimum arc of the graph.

Step 4: $\widetilde{M} \leftarrow \widetilde{M} \cup\left\{e_{i j}\right\}$

Step 5: end for

Step 6: $\widetilde{G^{\prime}} \leftarrow \widetilde{G}$ with all adges in $\widetilde{M}$ contracted

Step 7: Recursively compute the $\widetilde{M}$ till the size of $\widetilde{M}$ is $\left|V_{\widetilde{G}}\right|-1$ and $\left|V_{\widetilde{G}}\right|=1$ after edge contractions

Step 8: Calculate $\widetilde{W}(\widetilde{M})$ and defuzzify its value.

\section{An application IMST in Water Supply Network}

Water is the source of life, and is closely related to human survival. Water supply network is a water distribution system created by people, which is a vital part of water supply system. Water supply system in the order usually consists of water intake structures, water treatment structures, water supply pumping stations, adjustment structures, drainage pipes and water supply pipe network. The water supply network mainly refers to the urban water supply pipe network system, which is an important material base to protect the city people's life 
and develop production and construction. Urban water supply pipe network system can be regarded as an important lifeline of urban engineering. The traditional view is that the water supply network's mission is to provide sufficient amount of water, the residents have enough water to use. However, with the continuous development of social economy, people put forward higher requirements for the safety, reliability and economy of the water supply. Specifically, in the process of water supply, the quality of supplied water is healthy or not, such as water pipe corrosion or other factors lead to water quality problems; after an earthquake or major disaster, the water supply network is reliable or not, urban water supply network can be normal without the occurrence of secondary disasters; pipe network not only to have above two characteristics, economic issues is the focus. In the case of the entire water supply network connectivity, we must to ensure the lowest costs that aim to produce hedge-fund-like returns at lower cost. So in recent years, research on the economic aspect of water supply network is increasingly attracted people's attention. Water supply system is an important infrastructure of the city, and it also is an important part of urban lifeline project, which plays an irreplaceable role in protecting economic development, ensuring social production and meeting human life. In laying city network, the street interchanges must be considered because the pipelines must along the street to lay. In case of that water supply network can connect all users to make it with the lowest costs. To achieve this goal, you must consider how to select and handle these interchanges.

\subsection{Graphical Model for Water Supply Network}

In laying city nework, the street interchanges must be considered because the pipelines must along the street to lay. In case of that water supply network can connect all users to make it with the lowest costs. To achieve this goal, you must consider how to select and handle these interchanges. This problem is a serious problem. The solution of this problem can provide a standard for the laying of water supply network to ensure best desing effect. According to graph theory, the water supply network can be viewed as a graph. So the contents of the water supply network need to be translated into the language of graph theory to help solve the problem of water supply network costs. The language from pipelines to graph is described as follows :

1. The water supply center and users in the planning area are referred to as nodes, the intersection of the street known as the intersections. The nodes and intersections are regarded as the vertices of graph. So the issue can be converted to the shortest path between each vertex, and each vertex must be connected indirectly or not indirectly.

2. The routes that may be laying between nodes and intersections can be considered as edges of the graph.

3. The sum of construction costs and operating costs of each line is regarded as the weights of edges. The sum of weights is the minimum that is the lowest costs, that is the purpose of the design you want to achieve.

Through the above three steps, the water supply network can form a graph, this graph includes the vertices, edges and weights. Using $G=\left(V_{G}, E_{G}, W_{G}\right)$ to represent, $V_{G}$ represents the set of vertices in the graph; $E_{G}$ represents the set of edges in the graph; $W_{G}$ represents the set of weights of each edge in the graph. Setting $T_{G}$ is a spanning tree of diagram of $G$, then:

$$
W(T)=\sum_{e_{\alpha \beta}} W_{\alpha \beta}
$$


Among them, $W\left(T_{G}\right)$ is the sum of weights in the tree of $T_{G}$; euv is the any edge in the tree of $T_{G}$; Wuv is the weights of euv. The purpose of design is to require the minimum values of $W\left(T_{G}\right)$. Only this way can ensure the lowest costs of laying pipelines. In summary, the problem of the minimum costs of the water supply network may be as a problem of seeking minimum spanning tree in the graph. The minimum spanning tree must exist. According to the actual situation, each node will certainly connected when laying water supply network, so there will be a minimum spanning tree certainly. There are a variety of algorithms to generate minimum spanning tree, such as Prim algorithm, Kruskal algorithm and simple algorithm and so on.

\subsection{Numerical example}

A city wants to lay the pipelines of water supply network, the lines show in 2. Here we consider the edge weight of the graph as TIFN's. In figure $2 x_{6}$ is the source of water supply. We find the MST of the graph by using Borivka's algorithm [6]. The edges depicted as 'black' lines in Fig. 3 determine the edges of the spanning tree.

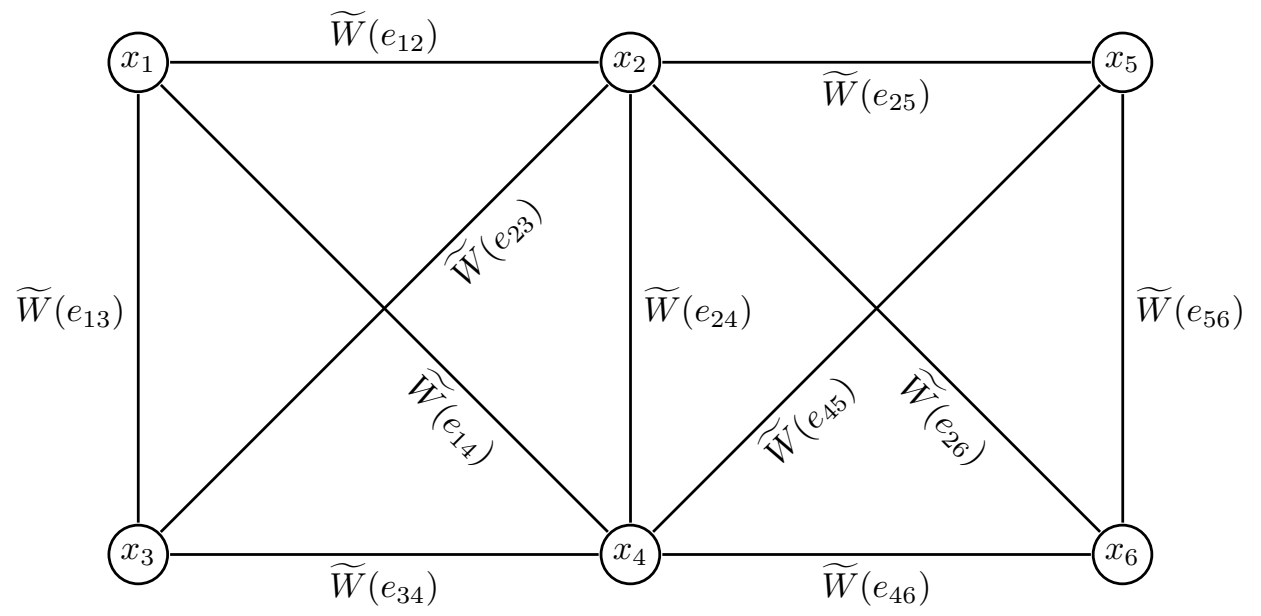

Figure 2: An UITFCG $\widetilde{G}$ whose edge weights are TIFN's

\section{Conclusion}

In this work, we proposed the intuitionistic fuzzy version of classical Borůva's algorithm to find the solution of IMST problem. The signification information for any decision makers in an IMST problem are the fuzzy minimum spanning tree and its corresponding cost. We can determine the IMST and its cost. We apply the mean of intuitionistic fuzzy numbers in our proposed algorithm to solve the problem. The main aim of this work is to propose an vague modeling of the MST problem. Intuitionistic fuzzy number can handle the uncertainties in edge weight of the edge to store the available information. In future study, we will study to use our introduced algorithmic approach to the real life scenarios like transportation, logistics, wireless communication and many other optimization problems that can be modeled as FMST problem and we will also try to improve the complexity of the proposed algorithm using fibonacci heap and adjacency list. 
Table 1: Edge Weights and score value of the Graph

\begin{tabular}{|c|c|c|}
\hline Edges & Intuitionistic Triangular Fuzzy Weight & Score value \\
\hline$\left(x_{1} x_{2}\right)$ & $\widetilde{W}\left(e_{12}\right)=([0.05,0.2,0.35] ; 0.2,0.5)$ & -0.021 \\
\hline$\left(x_{1} x_{3}\right)$ & $\widetilde{W}\left(e_{13}\right)=([0.2,0.35,0.7] ; 0.7,0.1)$ & 0.198 \\
\hline$\left(x_{1} x_{4}\right)$ & $\widetilde{W}\left(e_{14}\right)=([0.35,0.475,0.65] ; 0.5,0.5)$ & 0 \\
\hline$\left(x_{2} x_{4}\right)$ & $W\left(e_{24}\right)=([0.5,0.675,0.8] ; 0.3,0.6)$ & -0.069 \\
\hline$\left(x_{2} x_{3}\right)$ & $W\left(e_{23}\right)=([0.65,0.775,0.9] ; 0.4,0.5)$ & -0.035 \\
\hline$\left(x_{3} x_{4}\right)$ & $\widetilde{W}\left(e_{34}\right)=([0.8,0.95,1] ; 0.7,0.2)$ & 0.344 \\
\hline$\left(x_{2} x_{5}\right)$ & $W\left(e_{25}\right)=([0.2308,0.3,0.4286] ; 0.8,0.1)$ & 0.190 \\
\hline$\left(x_{5} x_{6}\right)$ & $\widetilde{W}\left(e_{56}\right)=([0.3,0.4286,0.75] ; 0.8,0.1)$ & 0.293 \\
\hline$\left(x_{4} x_{6}\right)$ & $W\left(e_{46}\right)=([0.55,0.7,0.85] ; 0.6443,0.252)$ & 0.191 \\
\hline$\left(x_{2} x_{6}\right)$ & $\widetilde{W}\left(e_{26}\right)=([0.7,0.8667,0.9667] ; 0.7846,0.1587)$ & 0.429 \\
\hline$\left(x_{4} x_{5}\right)$ & $\widetilde{W}\left(e_{45}\right)=([0.8,1,1] ; 0.8413,0.126)$ & 0.539 \\
\hline
\end{tabular}

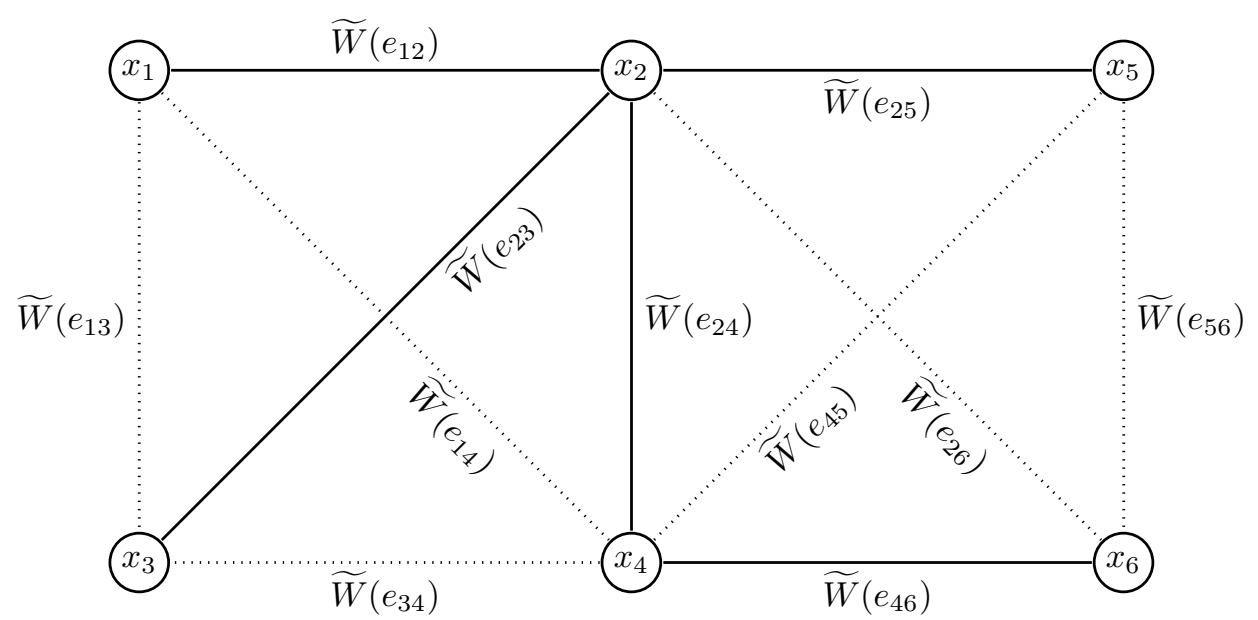

Figure 3: MST of $\widetilde{G}$

\section{References}

[1] H. Mao, L. W. Xie, Z. X. Zhang, Applying Pruning-based Minimum Spanning Tree Algorithm Water Supply Networks, Computer Applications and Software, 28, (2011), 109-110.

[2] A. Dey, A. Pal, Prim's algorithm for solving minimum spanning tree problem in fuzzy environment, Annals of Fuzzy Mathematics and Informatics, 12(3), (2016), 409-430.

[3] A. Dey, S. Broumi, L. H. Son, A. Bakali, M. Talea, F. Smarandache, A new algorithm for finding minimum spanning trees with undirected neutrosophic graphs, Granular Computing (Springer), 4(1), (2019), 63-69.

[4] A. Dey, R. Pradhan, A. Pal, T. Pal, A Genetic Algorithm For Solving Fuzzy Shortest Path Problems With Interval Type-2 Fuzzy Arc Lengths, Malaysian Journal of Computer Science, 31(4), (2018). 
[5] A. Dey, L. Son, A. Pal, Fuzzy Minimum Spanning Tree with Interval Type 2 Fuzzy Arc Length: Formulation and A New Genetic Algorithm, Soft computing, (2019), 1-12.

[6] J. Nešetřil, E. Milkovś, H. Nešetřilová and O. Borůvka Otakar Borůvka on minimum spanning tree problem Translation of both the 1926 papers, comments, history, Discrete Mathematics,, 233, (2001), 3-36.

[7] K. Atanassov, Intuitionistic fuzzy sets, Fuzzy sets and system, 20, (1986), 87-96.

[8] S. Das, S. Kar, T. Pal, Robust decision making using intuitionistic fuzzy numbers, Journal of New Theory, 2(1), (2017), 41-54.

[9] L.A. Zadeh, Fuzzy sets, Information and Control, 8, (1965), 338-353.

[10] M. G. Karunambigai, O. Kalaivani, Intuitionistic fuzzy graph, World Applied Science Journal, 14(12),(2011), 1928-1936.

[11] B. Asady and A. Zendehnam, Ranking fuzzy numbers by distance minimization, Applied Mathematical Modelling 31(11) (2007) 2589-2598. 176 (16) (2006) 2405-2416.

[12] S. Abbasbandy and T. Hajjari, A new approach for ranking of trapezoidal fuzzy numbers,Computers \& Mathematics with Applications 57(3) (2009) 413-419.

[13] T. A. De Almeida, A. Yamakami, and M. T. Takahashi, An evolutionary approach to solve minimum spanning tree problem with fuzzy parameters, in null. IEEE, (2005) 203-208.

[14] C. C. Chou, The canonical representation of multiplication operation on triangular fuzzy numbers, Computers \& Mathematics with Applications 45(10)(2003) 1601-1610.

[15] P. T. Chang and E. Lee, Fuzzy decision networks and deconvolution, Computers \& Mathematics with Applications, 37(11) (1999) 53-63.

[16] D. Dubois and H. Prade, Operations on fuzzy numbers, International Journal of systems science, 9(6) (1978) 613-626.

[17] J. C. Gower and G. Ross, Minimum spanning trees and single linkage cluster analysis, Applied statistics, (1969) 54-64.

[18] J. Gao and M. Lu, Fuzzy quadratic minimum spanning tree problem, Applied Mathematics and Computation, 164(3) (2005) 773-788.

[19] R. L. Graham and P. Hell, On the history of the minimum spanning tree problem, Annals of the History of Computing, 7 (1) (1985) 43-57.

[20] H. Ishii and T. Nishida, Stochastic bottleneck spanning tree problem, Networks, 13(3) (1983) 443-449.

[21] H. Ishii and T. Matsutomi, Confidence regional method of stochastic spanning tree problem, Mathematical and computer modelling, 22(10) (1995) 77-82.

[22] H. Ishii, S. Shiode, T. Nishida, and Y. Namasuya, Stochastic spanning tree problem, Discrete Applied Mathematics, 3(4) (1981) 1400-1412.

[23] T. Itoh and H. Ishii, An approach based on necessity measure to the fuzzy spanning tree problems, Journal of the Operations Research Society of Japan, 39(2) (1996) 247-257.

[24] A. Janiak and A. Kasperski, The minimum spanning tree problem with fuzzy costs, Fuzzy Optimization and Decision Making, 7(2) (2008) 105-118.

[25] J. B. Kruskal, On the shortest spanning subtree of a graph and the traveling salesman problem, Proceedings of the American Mathematical society 7(1) (1956) 48-50.

[26] A. N. C. Kang, R. C. Lee, C. L. Chang, and S. K. Chang, Storage reduction through minimal spanning trees and spanning forests,

[27] B. Liu and B. Liu, Theory and practice of uncertain programming, Springer, 2002.

[28] B. Liu, Uncertainty theory: An introduction to its axiomatic foundations, 2004.

[29] H. M. Neha and H. Hajmohamadi, A ranking function method for solving fuzzy multi-objective linear programming problem, Annals of Fuzzy Mathematics and Informatics 3(1) (2012) 31-38.

[30] R. E. Osteen and P. Lin, Picture skeletons based on eccentricities of points of minimum spanning 
trees, SIAM Journal on Computing, 3(1) (1974) 23-40.

[31] G. Rodolakis, A. Laouiti, P. Jacquet, and A. M. Naimi, Multicast overlay spanning trees in ad hoc networks: Capacity bounds, protocol design and performance evaluation, Computer Communications 31(7) (2008) 1400-1412.

[32] Salim Rezvani, Ranking method of trapezoidal intuitionistic fuzzy numbers, Annals of Fuzzy Mathematics and Informatics 5(3) (2013) 515-523.

[33] J. Zhou, L. Chen and K. Wang, Path optimality conditions for minimum spanning tree problem with uncertain edge weights, International Journal of Uncertainty, Fuzziness and Knowledge-Based Systems, 23(01) (2015) 49-71.

[34] J. Zhou, Q. Wang and X. Zhang, The inverse spanning tree of a fuzzy graph based on credibility measure, Journal of Communications, 8(9), (2013), 566-571. 